\title{
ON GENERALIZED MAXIMAL FUNCTIONS
}

\author{
BERND S. W. SCHRÖDER
}

(Communicated by J. Marshall Ash)

\begin{abstract}
In this paper we study the question of under what circumstances the quantity $\left\|\sup _{t<\infty, a \in \mathbf{R}}\left|\int_{0}^{t} f\left(a, M_{s}\right) d M_{s}\right|\right\|_{p}$ is comparable to $\left\|M_{\infty}^{*}\right\|_{p}$, where $M_{t}$ is a continuous martingale and $f$ is a bounded Borel-measurable function.
\end{abstract}

\section{INTRODUCTION}

Let $\left\{M_{t}\right\}_{t \geq 0}$ be a continuous martingale that starts at 0 and satisfies the "usual conditions". Let $M_{t}^{*}:=\sup _{s \leq t}\left|M_{s}\right|$ be the usual maximal function and let $L_{t}^{*}:=\sup _{a \in \mathbf{R}} L_{t}^{a}$ be the maximal function of the local time $L_{t}^{a}:=$ $\left(M_{t}-a\right)^{+}-\left(M_{0}-a\right)^{+}-\int_{0}^{t} 1_{M_{s}>a} d M_{s}$ of $M_{t}$. Barlow and Yor showed in $[2,3]$ that for all $p>0$ we have that $\left\|M_{\infty}^{*}\right\|_{p}$ is comparable to $\left\|L_{\infty}^{*}\right\|_{p}$, i.e., that there are universal constants $c, C>0$ only depending on $p$ such that $c\left\|M_{\infty}^{*}\right\|_{p} \leq\left\|L_{\infty}^{*}\right\|_{p} \leq C\left\|M_{\infty}^{*}\right\|_{p}$. It can be shown that for $p>0$ we have that $\left\|\sup _{t<\infty, a \in \mathbb{R}}\left|\int_{0}^{t} 1_{M_{s}>a} d M_{s}\right|\right\|_{p}$ is comparable to $\left\|M_{\infty}^{*}\right\|_{p}$. This immediately leads to the following question: Let $f: \mathbb{R}^{2} \rightarrow \mathbb{R}$ be a bounded Borel-measurable function. Under what circumstances is $\left\|\sup _{t<\infty, a \in \mathbb{R}}\left|\int_{0}^{t} f\left(a, M_{s}\right) M_{s}\right|\right\|_{p}$ comparable to $\left\|M_{\infty}^{*}\right\|_{p}$ ? We will give a sufficient condition (cf. Theorem 2.3) for this to happen, which enables us to give a wide variety of examples.

\section{RESULTS}

In this section we define a generalized maximal function for a continuous martingale (cf. Definition 2.1) and investigate when norm inequalities of the proposed type hold. In Theorem 2.3(i) we give a condition that ensures that the generalized maximal function is progressively measurable. Part (ii) is a lemma that also enables us to give the variety of examples as shown in Example 2.4. In part (iii) we give a sufficient condition for "upper comparability" that-in light of Example 2.5-appears to be fairly sharp. Finally, in part (iv) we give a sufficient condition for "lower comparability".

Received by the editors October 7, 1991; part of the present material has been presented at the AMS meeting in Baltimore, January 1992, and at the Mini-Conference on Harmonic Analysis and Probability Theory at Kansas State University, March 1992.

1991 Mathematics Subject Classification. Primary 60G46, 60H05, 42A61.

Key words and phrases. Martingale, local time, maximal function.

The author was partially funded by the Office of Naval Research under Contract N00014-88-K0455 . 
Definition 2.1. Let $f: \mathbb{R}^{2} \rightarrow \mathbb{R}$ be a bounded Borel-measurable function. Then we can define

$$
M f_{t}^{a}:=\int_{0}^{t} f\left(a, M_{s}\right) d M_{s}
$$

and we define the generalized maximal function of $M_{t}$ associated with $f$ to be

$$
M f_{t}^{*}:=\operatorname{ess} \sup _{s \leq t, a \in \mathbf{R}}\left|M f_{s}^{a}\right| .
$$

When there could be doubt as to which martingale a generalized maximal function belongs to we will write $M f\left(M_{u}\right)_{t}^{a}$ for $M f_{t}^{a}$, etc.

Definition 2.2. Let $\hat{p}>0$. Then $f$ satisfies the $\hat{p}$-continuity condition iff there is a $c>0$ such that for all $a, b \in \mathbb{R}$ we have that

$$
\int_{\mathbf{R}}|f(a, x)-f(b, x)|^{\hat{p}} d x \leq c|b-a| .
$$

$f$ satisfies the cone condition iff there is a $C>0$ such that for all $a \in \mathbb{R}$ we have that

$$
V_{-a}^{a} f(a, \cdot) \leq C,
$$

where $V_{-a}^{a} f(a, \cdot)$ denotes the variation of the function $x \mapsto f(a, x)$ over the interval $[-a, a]$.

Theorem 2.3. Let $\hat{p} \geq 2$ and let $f: \mathbb{R}^{2} \rightarrow \mathbb{R}$ be a bounded Borel-measurable function that satisfies the $\hat{p}$-continuity condition. Then

(i) $M f_{t}^{a}$ has a continuous version, i.e., there is a process $\widehat{M} f_{t}^{a}$ that is continuous in $(a, t)$ such that $\widehat{M} f_{t}^{a}=M f_{t}^{a}$ a.s. Therefore $M f_{t}^{*}$ is progressively measurable.

(ii) $g(a, x):=f(a, x) 1_{|a|<|x|}$ satisfies the $\hat{p}$-continuity condition and the cone condition.

(iii) If $f$ satisfies the cone condition, then for $0<p<\infty$ there is a $C_{p}>0$ such that for all continuous martingales $M_{t}$ we have that

$$
\left\|M f_{\infty}^{*}\right\|_{p} \leq C_{p}\left\|M_{\infty}^{*}\right\|_{p} .
$$

(iv) If there is an $\varepsilon>0$ such that for each $N>0$ there is an $a \in \mathbb{R}$ such that $\left.f(a, \cdot)\right|_{[-N, N]}>\varepsilon$, then for $0<p<\infty$ there is a $c_{p}>0$ such that for all continuous martingales $M_{t}$ we have that

$$
\left\|M f_{\infty}^{*}\right\|_{p} \geq c_{p}\left\|M_{\infty}^{*}\right\|_{p} .
$$

Example 2.4. Let $\hat{p} \geq 2$ and let $f: \mathbb{R}^{2} \rightarrow \mathbb{R}$ be a bounded Borel-measurable function that satisfies the $\hat{p}$-continuity condition. Let $\tilde{f}:=f(a, x) 1_{|a|<|x|}+$ $1_{a \leq-|x|}$. Then for $0<p<\infty$ we have that there are constants $c_{p}, C_{p}>0$ such that for all continuous martingales $M_{t}$ :

$$
c_{p}\left\|M_{\infty}^{*}\right\|_{p} \leq\left\|M \tilde{f}_{\infty}^{*}\right\|_{p} \leq C_{p}\left\|M_{\infty}^{*}\right\|_{p} .
$$

Example 2.5. Let

$$
\begin{aligned}
\mathscr{F}:=\{g: \mathbb{R} \rightarrow\{-1,0,1\} \mid \exists n \in \mathbb{N}: \forall k \in\{-n+1, \ldots, n\}: g \text { is constant } \\
\text { and } \left.|g|=1 \text { on }\left[\frac{k-1}{n}, \frac{k}{n}\right), \text { and }\left.g\right|_{\mathbb{R} \backslash[-1,1)}=0\right\} .
\end{aligned}
$$


Let $\left\{g_{n}\right\}_{n \in \mathbb{N}}$ be a counting of $\mathscr{F}$. Define

$$
f(a, x):= \begin{cases}g_{a / 2}(x) & \text { if } a \in \mathbb{N} \text { is even } \\ 0 & \text { if } a \in \mathbb{N} \text { is odd or } a \leq 0 \\ (1-(a-[a])) f([a], x) & +(a-[a]) f([a]+1, x) \quad \text { otherwise }\end{cases}
$$

Then $f$ satisfies the $\hat{p}$-continuity condition for $\hat{p}=2$ and it obviously does not satisfy the cone condition. In can be shown (cf. [7]) that $M f_{t}^{*}=\infty$ on a set of positive measure for $t>0$ and for $M_{t}$ Brownian motion stopped at a time $T>0$.

\section{THE PROOF}

Throughout this proof let $M_{t}$ be a continuous martingale and let $f: \mathbb{R}^{2} \rightarrow \mathbb{R}$ be a bounded Borel-measurable function that satisfies the $\hat{p}$-continuity condition for some $\hat{p} \geq 2$. Let $\hat{q}$ be such that $1 / \hat{p}+1 / \hat{q}=1$, and let $T>0$. Recall that for $x>0$ we have that $\tau_{x}:=\inf \left\{t>0:\left|M_{t}\right|=x\right\}$ is a stopping time.

(a) $M f_{t}^{a}$ has a version $\widehat{M} f_{t}^{a}$ that is jointly continuous in $(a, t)$. Thus $M f_{t}^{a}$ is progressively measurable and since $M f_{t}^{*}=\widehat{M} f_{t}^{*}$ a.s. we are free to assume without loss of generality that $M f_{t}^{a}$ is continuous. Moreover there is a constant $C>0$ depending on $\hat{p}$ and $p^{\prime} \geq \hat{q}$ only such that for all $\lambda>0$, and $r, s \in \mathbb{R}$ we have that

$$
E\left(\sup _{t \leq T}\left|M f_{t}^{r}-M f_{t}^{s}\right|^{p^{\prime}} ; M_{T}^{*} \leq \lambda\right) \leq C|r-s|^{p^{\prime} / \hat{p}} E\left(\left(M_{T}^{*} \wedge \lambda\right)^{p^{\prime} / \hat{p}}\right) .
$$

An application of [2, Theorem 4.1] shows that

$$
\begin{aligned}
\left\|\sup _{t \leq T}\left|M f_{t}^{a}-M f_{t}^{b}\right|\right\|_{p^{\prime}} \\
\leq C^{\prime}\left\|\left\langle\int_{0}^{\cdot}\left(f\left(a, M_{t}\right)-f\left(b, M_{t}\right)\right) d M_{t}\right\rangle_{T}^{1 / 2}\right\|_{p^{\prime}} \\
\leq C^{\prime \prime}\|f(a, \cdot)-f(b, \cdot)\|_{\hat{p}\left\|\langle M\rangle_{T}^{1 / 2}\right\|_{p^{\prime} / \hat{q}}^{1 / q} \quad(\text { by [2, Theorem 4.1]) }} \\
\leq C^{\prime \prime \prime}|b-a|^{1 / \hat{p}}\left\|\langle M\rangle_{T}^{1 / 2}\right\|_{p^{\prime} / \hat{q}}^{1 / \hat{q}} .
\end{aligned}
$$

To prove the assertions regarding continuity, let $p^{\prime}>\hat{p}, \hat{q}$ and let $N>0$. Clearly $\left\|\langle M\rangle_{t \wedge \tau_{N}}\right\|_{p^{\prime} / \hat{q}}<\infty$. Thus there is a constant $\bar{C}>0$ such that

$$
E\left(\sup _{s \leq t}\left|M f_{s \wedge \tau_{n}}^{a}-M f_{s \wedge \tau_{n}}^{b}\right|^{p^{\prime}}\right) \leq \bar{C}|b-a|^{p^{\prime} / \hat{p}}
$$

An application of Kolmogorov's Theorem on continuous processes now yields that $M f_{t \wedge \tau_{N}}^{a}$ has a jointly continuous version. Since $\tau_{N} \rightarrow \infty$ for $N \rightarrow \infty$ this implies that $M f_{t}^{a}$ has a jointly continuous version. An easy computation now shows that $M f_{t}^{*}$ is progressively measurable and $M f_{t}^{*}=\widehat{M} f_{t}^{*}$ a.s. 
Finally for $t \leq T$ on $\left\{M_{T}^{*} \leq \lambda\right\}=\left\{T \leq \tau_{\lambda}\right\}$ we a.s. have that

$$
\begin{aligned}
M f\left(M_{u}\right)_{t}^{r} & =\int_{0}^{t} f\left(r, M_{s}\right) d M_{s}=\int_{0}^{t \wedge \tau_{\lambda}} f\left(r, M_{s \wedge \tau_{\lambda}}\right) d M_{s \wedge \tau_{\lambda}} \\
& =\int_{0}^{t} f\left(r, M_{s \wedge \tau_{\lambda}}\right) d M_{s \wedge \tau_{\lambda}}=M f\left(M_{u \wedge \tau_{\lambda}}\right)_{t}^{r},
\end{aligned}
$$

and therefore

$$
\begin{aligned}
& E\left(\sup _{t \leq T}\left|M f\left(M_{u}\right)_{t}^{r}-M f\left(M_{u}\right)_{t}^{s}\right|^{p^{\prime}} ; M_{T}^{*} \leq \lambda\right) \\
& \quad \leq E\left(\sup _{t \leq T}\left|M f\left(M_{u \wedge \tau_{\lambda}}\right)_{t}^{r}-M f\left(M_{u \wedge \tau_{\lambda}}\right)_{t}^{s}\right|^{p^{\prime}}\right) \\
& \quad \leq C|r-s|^{p^{\prime} / \hat{p}} E\left(\left(M_{T \wedge \tau_{\lambda}}^{*}\right)^{p^{\prime} / \hat{q}}\right) \quad(\text { by }(*)) \\
& \quad=C|r-s|^{p^{\prime} / \hat{p}} E\left(\left(M_{T}^{*} \wedge \lambda\right)^{p^{\prime} / \hat{q}}\right) .
\end{aligned}
$$

(b) Suppose that $f$ is such that for $|a| \geq|x|$ we have that $f(a, x)=0$. Then for all $\lambda>0$ there is a nullset $S \subseteq\left\{M_{T}^{*} \leq \lambda\right\}$ such that for all $\omega \in\left\{M_{T}^{*} \leq \lambda\right\} \backslash S$ we have that $M f_{t}^{r}=0$ for $|r| \geq \lambda$.

First notice that by continuity of $M f_{t}^{r}$ it is enough to prove that $M f_{t}^{r}=0$ for $|r| \geq \lambda$ with $t, r \in \mathbb{Q}$. For $t \leq T$ and $|r| \geq \lambda$ there is a nullset $S_{r, t}$ such that for $\omega \in\left\{M_{T}^{*} \leq \lambda\right\} \backslash S_{r, t}$ :

$$
M f_{t}^{r}=M f_{t \wedge \tau_{\lambda}}^{r}=\int_{0}^{t \wedge \tau_{\lambda}} f\left(r, M_{u}\right) d M_{u}=\int_{0}^{t \wedge \tau_{\lambda}} 0 d M_{u}=0 .
$$

Thus by continuity of $M f_{t}^{r}$ we have on $\left\{M_{T}^{*} \leq \lambda\right\} \backslash \bigcup_{|r| \geq \lambda, r \in \mathbb{Q}, t \leq T, t \in \mathbb{Q}} S_{r, t}$ that $M f_{t}^{r}=0$ for $|r| \geq \lambda$.

(c) Suppose that $f$ is such that for $|a| \geq|x|$ we have that $f(a, x)=0$. Then there is a $C>0$ depending only on $p$ such that

$$
\left\|M f_{\infty}^{*}\right\|_{p} \leq C\left\|M_{\infty}^{*}\right\|_{p} .
$$

This part of the proof was inspired by the work of Gundy and Silverstein in [5]. It is completely analogous to the conclusion of the proof in [5, §4]. Recall from [5] the following version of the Garsia-Rodemich-Rumsey-Lemma (GRR): If a continuous function $F: I=[a, b] \rightarrow \mathbb{R}$ has a zero in $I$, then for $q>0$, $p^{\prime}>\frac{2}{q}$ we have that

$$
\sup _{r \in I}|F(r)| \leq C|I|^{q}\left\{\frac{1}{|I|^{2}} \iint_{I \times I}\left|\frac{F(r)-F(s)}{|r-s|^{q}}\right|^{p^{\prime}} d r d s\right\}^{1 / p^{\prime}}
$$

where $C$ is a universal constant.

Also recall from [5] that if there are $p^{\prime}>p$ and $C, D>0$ such that for all $\lambda>0$ we have that

$$
P(G \geq \lambda) \leq \frac{C}{\lambda p^{\prime}} \int_{\{H \leq \lambda\}} H^{p^{\prime}} d P+D P(H>\lambda),
$$

then

$$
\int_{\Omega} G^{p} d P \leq\left(C \frac{p}{p^{\prime}-p}+D\right) \int_{\Omega} H^{p} d P
$$


Let $p^{\prime}>\max \{2 \hat{p}, p \hat{q}\}$ and let $S$ be as in (b). By GRR with $I:=[-\lambda, \lambda]$ for $\omega \in\left\{M_{T}^{*} \leq \lambda\right\} \backslash S$ we have that

$$
\begin{aligned}
\left(M f_{T}^{*}\right)^{p^{\prime}} & =\sup _{t \leq T} \sup _{|r| \leq \lambda}\left|M f_{t}^{r}\right|^{p^{\prime}} \quad(\text { by }(\mathrm{b})) \\
& \leq \sup _{t \leq T} C \lambda^{p^{\prime} / \hat{p}} \frac{1}{(2 \lambda)^{2}} \int_{I \times I}\left(\frac{\left|M f_{t}^{r}-M f_{t}^{s}\right|}{|r-s|^{1 / \hat{p}}}\right)^{p^{\prime}} d r d s \quad \text { (by GRR) } \\
& \leq C \lambda^{p^{\prime} / \hat{p}} \frac{1}{(2 \lambda)^{2}} \int_{I \times I}\left(\frac{\sup _{t \leq T}\left|M f_{t}^{r}-M f_{t}^{s}\right|}{|r-s|^{1 / \hat{p}}}\right)^{p^{\prime}} d r d s .
\end{aligned}
$$

Integration now yields via (a)

$$
E\left(\left(M f_{T}^{*}\right)^{p^{\prime}} ; M_{T}^{*} \leq \lambda\right) \leq C^{\prime} \lambda^{p^{\prime} / \hat{p}} E\left(\left(M_{T}^{*} \wedge \lambda\right)^{p^{\prime} / \hat{q}}\right) .
$$

Therefore by Chebyshev's inequality:

$$
\begin{aligned}
P\left(M f_{T}^{*}>\lambda\right) & \leq P\left(M f_{T}^{*}>\lambda, \quad M_{T}^{*} \leq \lambda\right)+P\left(M_{T}^{*}>\lambda\right) \\
& \leq \frac{1}{\lambda p^{\prime}} E\left(\left(M f_{T}^{*}\right)^{p^{\prime}} ; M_{T}^{*} \leq \lambda\right)+P\left(M_{T}^{*}>\lambda\right) \\
& \leq \frac{C^{\prime}}{\lambda p^{\prime}(1-1 / \hat{p})} E\left(\left(M_{T}^{*} \wedge \lambda\right)^{p^{\prime} / \hat{q}}\right)+P\left(M_{T}^{*}>\lambda\right) \\
& \leq \frac{C^{\prime}}{\lambda p^{\prime} / \hat{q}} E\left(\left(M_{T}^{*}\right)^{p^{\prime} / \hat{q}} ; M_{T}^{*} \leq \lambda\right)+\left(C^{\prime}+1\right) P\left(M_{T}^{*}>\lambda\right)
\end{aligned}
$$

Using the above mentioned good- $\lambda$-inequality we obtain

$$
E\left(\left(M f_{T}^{*}\right)^{p}\right) \leq C^{\prime \prime} E\left(\left(M_{T}^{*}\right)^{p}\right) .
$$

(d) For the usual local time $L_{t}^{a}=\left(M_{t}-a\right)^{+}-\left(M_{0}-a\right)^{+}-\int_{0}^{t} 1_{M_{s}>a} d M_{s}$ of $M_{t}$ and $p>0$ there is a $C_{p}>0$ that only depends on $p$ such that

$$
\left\|L_{T}^{*}\right\|_{p} \leq C_{p}\left\|M_{T}^{*}\right\|_{p}
$$

Clearly

$$
L_{T}^{*} \leq\left(\left(M_{t}-a\right)^{+}-\left(M_{0}-a\right)^{+}\right)_{T}^{*}+\left(\int_{0}^{t} 1_{M_{s}>a} d M_{s}\right)_{T}^{*},
$$

where $X(t, a)_{T}^{*}=\sup _{t \leq T, a \mathbf{R}}|X(t, a)|$. Moreover

$$
\left(\left(M_{t}-a\right)^{+}-\left(M_{0}-a\right)^{+}\right)_{T}^{*} \leq M_{T}^{*} .
$$

To see that there is a constant $C>0$ only depending on $p$ such that

$$
\left\|\left(\int_{0}^{t} 1_{M_{s}>a} d M_{s}\right)_{T}^{*}\right\|_{p} \leq C\left\|M_{T}^{*}\right\|_{p}
$$

notice that part (c) works for $f(a, x):=1_{x>a}$ since this function satisfies the $\hat{p}$-continuity condition for any $\hat{p}>0$ and since with arguments as in (b) one can show that on $\left\{M_{T}^{*} \leq \lambda\right\}$ we a.s. have that $M\left(1_{x>a}\right)_{t}^{r}=0$ for $r \geq \lambda$ and $M\left(1_{x>a}\right)_{t}^{r}=M_{t}$ for $r \leq-\lambda$. Thus the above also provides a new short proof of the result of Barlow and Yor. 
(e) Let $g(a, x):=f(a, x) 1_{|a|<|x|}$ and let $h(a, x):=f(a, x) 1_{|a| \geq|x|}$. Then $g$ and $h$ satisfy the $\hat{p}$-continuity condition.

We only give the proof for $h$, since the proof for $g$ is similar. Let $f$ be bounded by, say, $C$, let $a, b \in \mathbb{R}$ be fixed, and let $a \leq b$. Then

$$
\begin{aligned}
\|h(a, \cdot)-h(b, \cdot)\|_{\hat{p}} \\
\quad \leq \| f(a, \cdot) 1_{|a| \geq|\cdot|}-f(b, \cdot) 1_{|a| \geq \mid \cdot \cdot\left\|_{\hat{p}}+\right\| f(b, \cdot) 1_{|a| \geq|\cdot|}-f(b, \cdot) 1_{|b| \geq \mid \cdot \cdot \|_{\hat{p}}}} \\
\quad=\|(f(a, \cdot)-f(b, \cdot)) 1_{|a| \geq|\cdot| \cdot\left\|_{\hat{p}}+\right\| f(b, \cdot)\left(1_{|a| \geq|\cdot|}-1_{|b| \geq|\cdot|)}\right) \|_{\hat{p}}} \\
\quad \leq\|f(a, \cdot)-f(b, \cdot)\|_{\hat{p}}+C\left\|\left(1_{|a| \geq|\cdot|}-1_{|b| \geq|\cdot|)}\right)\right\|_{\hat{p}} \leq C^{\prime}|b-a|^{1 / \hat{p}} .
\end{aligned}
$$

(f) Suppose that $f$ satisfies the cone condition and let $h$ be as in (e). Then there is a constant $C_{p}>0$ that only depends on $p$ such that

$$
\left\|M h_{T}^{*}\right\|_{p} \leq C_{p}\left\|M_{T}^{*}\right\|_{p} .
$$

An application of the Hô-Tanaka formula shows that for all $a \in \mathbb{R}$ :

$$
\frac{1}{2} \int_{\mathbf{R}} L_{t}^{x} d h(a, x)=\int_{M_{0}}^{M_{t}} h(a, y) d y-\int_{0}^{t} h\left(a, M_{s}\right) d M_{s} \quad \text { a.s. }
$$

Therefore by the continuity of $M h_{t}^{r}$ we have that

$$
\begin{aligned}
M h_{T}^{*} & =\sup _{t \leq T, a \in \mathbf{R}}\left|\int_{0}^{t} h\left(a, M_{s}\right) d M_{s}\right| \\
& \leq \sup _{t \leq T, a \in \mathbf{R}}\left|\int_{M_{0}}^{M_{t}} h(a, y) d y\right|+\sup _{t \leq T, a \in \mathbf{R}}\left|\frac{1}{2} \int_{\mathbb{R}} L_{t}^{x} d h(a, x)\right| \\
& \leq \sup _{t \leq T, a \in \mathbf{R}}\left|\int_{M_{0}}^{M_{t}}\right| h(a, y)|d y|+\frac{1}{2} \sup _{a \in \mathbf{R}} V_{-a}^{a} h(a, \cdot) \sup _{t \leq T, a \in \mathbf{R}}\left|L_{t}^{x}\right| \\
& \leq C M_{T}^{*}+C L_{T}^{*} .
\end{aligned}
$$

Proof of Theorem 2.3. Parts (i) and (ii) are proved in (a) and (e), respectively. To prove part (iii) let $g$ and $h$ be as in (e). Since $g$ satisfies the hypothesis of (c) we have that there is a $C>0$ that only depends on $p$ such that $\left\|M g_{T}^{*}\right\|_{p} \leq$ $C\left\|M_{T}^{*}\right\|_{p}$. Combining this with (f) we obtain:

$$
\left\|M f_{T}^{*}\right\|_{p} \leq\left\|M g_{T}^{*}\right\|+\left\|M h_{T}^{*}\right\|_{p} \leq C_{p}\left\|M_{T}^{*}\right\|_{p} .
$$

Now let $T$ go to infinity.

To prove (iv) for $N>0$ let $a_{N}$ be such that $\left.f\left(a_{N}, \cdot\right)\right|_{[-N, N]}<\varepsilon$. Then

$$
\begin{gathered}
E\left(\sup _{a \in \mathbf{R}, t>0}\left|\int_{0}^{t} f\left(a, M_{s}\right) d M_{s}\right|^{p}\right) \geq E\left(\sup _{a \in \mathbf{R}, t>0}\left|\int_{0}^{t \wedge \tau_{N}} f\left(a, M_{s}\right) d M_{s}\right|^{p}\right) \\
\geq E\left(\left(\left(\int_{0}^{\cdot \wedge \tau_{N}} f\left(a_{N}, M_{s}\right) d M_{s}\right)_{\infty}^{*}\right)^{p}\right) \\
\geq C^{\prime} E\left(\left(\int_{0}^{\tau_{N}} f^{2}\left(a_{N}, M_{s}\right) d\langle M\rangle_{s}\right)^{p / 2}\right) \geq C^{\prime} \varepsilon^{2} E\left(\langle M\rangle_{\tau_{N}}^{p / 2}\right) .
\end{gathered}
$$

Now let $N$ go to infinity. 
Proof of Example 2.4. It is easy to see that $\tilde{f}$ satisfies the conditions in (iii) and (iv) of Theorem 2.3.

Proof of Example 2.5. The proof is tedious but not very hard and can be found in [7].

\section{Conclusion}

We have defined generalized maximal functions and shown that a wide variety of generalized maximal functions satisfy norm inequalities of the BarlowYor type. The results presented here complement the results that Bass and Davis obtained in [1, 4]. In [1, 4] it is shown that Barlow-Yor-like inequalities hold for functionals that have scaling properties similar to the scaling properties of local time, while our results show that Barlow-Yor-like results hold for generalized maximal functions $M f_{t}^{a}$ for which $f$ has geometric properties that are similar to the properties of $1_{x>a}$. It is worth pointing out that the results presented here are not accessible from the results in [1, 4].

An interesting problem that will be the object of our further research is how to define a generalized density functional of the area integral (cf. [5]).

Results on generalized maximal functions for semimartinagles, and martingales that do not start at 0 , and the relation of these results to the results presented in [1] can be found in [7]. In [7] we also consider the slightly more general case where $f$ need not be bounded.

\section{ACKNOWLEDGMENT}

The results presented here are part of my $\mathrm{Ph} . \mathrm{D}$. dissertation. I would like to thank my advisor A. Bennett for his constant encouragement and support during this endeavor.

\section{REFERENCES}

1. Richard Bass, $L_{p}$-inequalities for functionals of Brownian motion, Seminaire de Probabilites XXI, Lecture Notes in Math., vol. 1247, Springer-Verlag, Berlin and New York, 1987, pp. 206-217.

2. M. T. Barlow and M. Yor, (Semi-) martingale inequalities and local times, Z. Wahrsch. Verw. Gebiete 55 (1981), 237-254.

3. _ Semi-martingale inequalities via the Garsia-Rodemich-Rumsey Lemma and applications to local times, J. Funct. Anal. 49 (1982), 198-229.

4. Burgess Davis, On the Barlow-Yor inequalities for local time, Seminaire de Probabilites XXI, Lecture Notes in Math., vol. 1247, Springer-Verlag, Berlin and New York, 1987, pp. 218-220.

5. R. F. Gundy and M. L. Silverstein, The density of the area integral in $\mathbb{R}_{+}^{n+1}$, Ann. Inst. Fourier (Grenoble) 35 (1985), 215-229.

6. N. Ikeda and S. Watanabe, Stochastic differential equations and diffusion processes, NorthHolland/Kodansha, Tokyo, 1981.

7. B. S. W. Schröder, On generalized maximal functions and distorted local times, Ph.D. Thesis, Kansas State University, 1992.

Department of Mathematics, Kansas State University, Manhattan, Kansas 66506 Current address: Department of Mathematics, Hampton University, Hampton, Virginia 23668 E-mail address: miamee@unixvax.hamptonu.edu 